\title{
Artificial Unintelligence: Anti-Intelligence of Intelligent Algorithms *
}

\author{
Yuhong Zhang ${ }^{1,2}$ and Umer Nauman ${ }^{1}$ \\ 1 Information Science and Engineering College, Henan University of Technology, \\ Zhengzhou, 450001, China \\ 2 Network and Data Security Key Laboratory of Sichuan, University of Electronic \\ Science and Technology, Chengdu, 610054, China \\ zhangyuhong001@gmail.com, stormy.umer@gmail.com
}

\begin{abstract}
In the age of big data, artificial intelligence (AI) algorithms play an important role in demonstrating the value of data, but its negative effects are increasingly prominent. Researchers focus more on the obvious issues like privacy protection these days, and we assume that technologies like AI will make people wiser. But due to the not-so-obvious limits of AI, it may push us to the opposite side-artificial unintelligence. In this paper, a detailed analysis of the process of value creation from data is presented first. Then, based on the above process, the role played by AI algorithms is analyzed. Next, the anti-intelligence features of AI algorithms, such as the tendency of 'fatuous King', the generalization of quick thinking, a faster transformation of master/slave at the individual level, and retribalization at the group level, are deeply discussed. Finally, some possilbe ways of circumventing the aforementioned problems are offered.
\end{abstract}

Keywords: big data · artificial intelligence algorithm · quick thinking · master-slave transformation.

\section{Introduction}

We are in the era of big data. The value of big data has been confirmed to a large extent. Obviously, the value of it is not the data. Data itself is not the solution. It is just part of the path to that solution (say, the valuable insights hidden from the data) [1]. However, finding insights from the massive data is not easy. People are incapable of dealing with this issue directly due to the deficiencies in terms of memory and computational power. As a result, we often mechanize and engineer mental activities that humans are not good at, and then outsource them to machines. In simple terms, this kind of mental activity process in which

\footnotetext{
* This paper is partly supported by Henan Provincial Key Scientific and Technological Plan (no.152102210261, no. 162300410056) , Plan of Nature Science Fundamental Research in Henan University of Technology (no.2015QNJH17) and National Natural and Science Foundation of China (no. 61602154)
} 
machines replace humans can be collectively referred to as artificial intelligence (AI) algorithms.

With the help of AI algorithms, the value of big data is shining. However, value comes at its fair price, because there is no free lunch in the world. Therefore, we need to check carefully what kind of role AI algorithms play in the production chain of big data value.

Some ongoing researches have shown that the technologies including AI may not make us smarter. We have to pay the price for the 'lunch provided by AI algorithms. The price includes the tendency to be a 'fatuous king' in digital age, the generalization for quick thinking, the faster transformation between master and slavery at the individual level, whose idea proposed by Hegel, and retribalization at a group level. All of the secondary products of AI algorithms may create a dangerous situation of artificial unintelligence. We will discuss these topics separately in the rest of the paper.

\section{Value Creation from Big Data}

\subsection{Core value of big data}

The core value of big data is rooted in its ability to prediction [2]. When it comes to the value of big data, many people think of it as scientific value. But in fact, the scientific value of big data is only a by-product during realizing its commercial value. This point can be seen from how domestic and foreign famous big data companies (such as Alibaba, Google, Amazon, etc.) monetize their investments.

There are many Internet giants described above, which are typically big data companies. In terms of business model, they may vary widely. But in the way of monetizing big data, they all come together in the same way. Most of them can be classified as advertising technology companies since their key businesses are mostly based on computational advertising $(\mathrm{CA})$.

In $\mathrm{CA}$, the role of prediction is evident. There is a need to predict which user is interested in which advertisement, and then the corresponding advertisement is pushed. Herein we use a simple case to illustrate how the value of data is produced.

\subsection{Demonstration of data value}

In order to monetize their assets, Internet companies (namely, big data companies) can achieve it in two ways. One is the use of traffic. This approach means that, if people visit your website, or use your App, in addition to serving content that the user is interested in (mostly free), the website (or App) can also carry some sponsored contents (i.e., advertisements). The sponsored contents are carried in free ones, which is the basis for the monetization of traffic.

Obviously, the monetization of traditional traffic-based advertising is inefficient. This is because all of people will see the same advertisement. Accordingly, 
the ad conversion rate is expected to be too low since most of users have no feelings of these ads or even disgusted with them. For Internet companies, it is a big deal to boost the conversion rate. Actually, these companies have a very important magic weapon, which is data [3].

For example, suppose we have a website with 100,000 visits per day. Then we can place an ad space on it (for instance, razor advertising). This ad space can have a quotation, say $5,000 \$$, which is the value of the traffic monetization. Clearly, it has flaws in this way, since the effective audiences for razor advertising are basically men. In other words, about half of the audiences (female) are wasted. Now the question remains: how to rationally use the other half of traffic?

In fact, in computational advertising system, we can do exactly that, leaving only half of the traffic to this razor advertiser for men audience. For the advertiser, the reach of effective audience is not compromised. But because he only uses half of the traffic, search-ad platform can give him a discount, say 3,000\$.

As a result, Internet companies can sell the remaining half of the women's traffic to another advertiser who sells cosmetics at a price of $3,000 \$$. For advertiser, he/she should be satisfied with the result because he/she spends less money to get same effective audience $(5,000 \$-3,000 \$=2000 \$)$. As for the Internet companies, they earn more money than before $(3,000 \$+3,000 \$=6,000 \$)$.

There is no doubt that it is a win-win business. However, the win-win (or multi-win) situation is, to some extent, inconsistent with the principle of interests conservation. To ahead for a win-win situation, new resources must be injected into this process of profit distribution. What is this extra resource? It is data. In fact, Internet companies are able to get a premium of $1,000 \$$ just because they have got the gender data of each user. This is the value that data create.

\subsection{No free lunch theorem}

As previously analyzed, data indeed can create value. If we continue to crossexamine, why do Internet companies have such data? In fact, the reason is not complicated. To a large extent, it is because Internet users make use of their products for free. This is the price of the so-called "free". As we all know, "There is no free lunch in the world."

Many Internet companies offer a variety of Internet products (such as search engines, social networks, and e-commerce APPs). Most of these products are free. When users use these products, they leave behind clues so regardlessly. Internet companies collect this information (i.e., electronic imprinting) and form a user profile. A user profile is a collection of information associated with a user. It is the explicit digital representation of the identity of the user. The user profile helps in ascertaining the interactive behavior of the user along with preferences.

After obtaining the user profiles, Internet companies can use them to deliver related paid content or products. Naturally, such "targeted" or "catering to" promotion is much better than blind promotion without any information guidelines. 


\subsection{Role of Artificial Intelligence Algorithms}

The case listed above is relatively simple, but in a real business environment, the number of users, the complexity of data and the sorts of advertisement rise far above that of the simple case.

Computational advertising companies (i.e. big data companies) want to find the "best match" among particular users and the corresponding advertisement in a context. The biggest challenge facing this "best match" is the large-scale optimization and search problem under complex constraints. In such scenarios, if there is no AI algorithm heavily involved, it is impossible to efficiently complete the massive matching task.

Therefore, it can be said that AI algorithms play a very important role in extracting useful knowledge (namely, insights) from large amount of data. In the process of mining value from big data, researchers and the general public often show great concern about the fall of personal privacy.

But is it so simple? Of course not. Besides that, AI algorithms have aggravated its potentials of "anti-intellectual" in the digital age [4]. Next Let us put this issue below the fold.

\section{Anti-Intelligence Features of AI Algorithms}

\subsection{Tendency of 'fatuous King'}

With the help of AI algorithms, even if you are in a minority in any small scale, your preferences or opinions can be realized to some extent. Even if your viewpoint is wrong or your behavior is not in line with mainstream social values, it is not difficult to find your followers by collaborative filtering recommendation algorithm. Clearly, it will worsen the cognitive prejudice of the world around us, which gradually results in a modern version of the "fatuous king" in digital age. As we know, in ancient history, we usually called a ruler as "fatuous king", who only listened to one-sided story to make wrong decision.

This argument is supported by facts. For example, China's emerging news client giant, Jinri Toutiao (i.e., todays headlines), which is a Beijing-based news and information content platform, short video giants, Tik Tok (also known as Douyin), which is a Chinese music video platform and social network, and the US social giant Facebook, the users number of these companies are hundreds of millions, and their AI models will generate a tailored feed list of content (including news, videos, friends and even political ads ) for each user, by analyzing the features of content and user profiles.

The well-known big data scholar Zhipei Tu called this type of data a "data pill", "once you continue to take it, you subconsciously change your judgment of something." In this situation, it is very easy for us to fall into a narrow view. It seems that every choice is made by us. However, those things we do not like or prone to reject are all filtered by AI algorithms. Gradually, we will hold a misguided belief that the world we see is the entire world, and naturally become considerable arrogance. 
Therefore, one will face a disadvantageous scenario in which all the personalized services (including information) are provided by AI algorithms. There is no doubt that it will contribute us to be a "fatuous king" in AI age.

\subsection{Generalization of Quick Thinking}

From the analysis above, we can see that, due to information overload, one has to rely on the recommendation of personalized content. These seemingly good recommendations will strengthen our ability of quick thinking and weaken our ability of thinking slow.

The concepts of "thinking fast" and "thinking slow " are put forward by Daniel Kahneman in his book "Thinking, Fast and Slow" [5]. In this book, Kahneman explains the dichotomy between two modes of thought - "System 1" that is fast, instinctive and emotional; "System 2" that is slower, more deliberative, and more logical.

As for human decision-making, due to the laziness of the slow-thinking system (it will consume more energy), brain does not start it easily. As a result, most of the time, brain is dominated by quick thinking.

But quick thinking is flawed because it produces more cognitive bias. It means that, to achieve rapid decision-making, we have to repeatedly adopt a psychological shortcut heuristics (i.e, quick thinking).

In the era of AI, AI algorithms can provide us all kinds of decisions that look good. Due to highly engineered solutions provided by intelligent algorithm$\mathrm{s}$, even if our mind can switch to "slow thinking" model, finally we find that decisions which are made by machine are usually better than ours. Overtime, people tend to quit the thankless job, which results in weakening our capacity of "slow thinking" gradually. The serious consequence that followed is to generalize our ability of quick thinking.

If a further deduction is made, we will see that, the "free-will" of which human beings have been proud may become "pseudo-free", since all of choices seem to be our own, but in fact, they just are the derivative results that are recommended by AI algorithms.

\subsection{Retribalization of the digital age}

The famous media theorist and thinker Marshall McLuhan made a three-phase division of the development of human society: "tribalization - detribalization - retribalization." In the age of AI, the evolution of the three phases will be accelerated.

In simple terms, "tribalization" means that, starting from the primitive society, dispersed individuals need to build a network of collaborative relationships to form a relatively stable and small alliance, which is a natural community of interests and discourse. The tribes are isolated and closed from each other.

In Web 1.0, the essence of the Internet is connection. It realizes the online expression of the physical world and generates convergent information. Through the online connection, the "tribalization" of the online community is realized. 
In Web 2.0, the information began to flow interactively, and Internet flattened the world. In other words, each point can be easily interconnected. Even if users are separated from each other by thousands of miles, and the media still can push similar news, social media, which allows us to "get closer to the world (namely global village)". The closed tribe gradually begin to disintegrate. This process is called "detribalization."

Internet forecasters even believed that Internet would lead to a massive increase in "detribalization". However, decades have passed and the theory has not been applied yet. Instead, with the rise of Web 3.0, human beings do not realize the Great Unity. On the contrary, everyone seems to enter a small circle and is barely able to scrape himself off the floor.

The most significant feature of Web 3.0 is that information begin to "actively (intelligently)" find people. Through the personalized recommendation of AI algorithms, even if a very small group of "individuals" can become "groups" again. Interconnecting technologies make it easier for us to find our own memes, so that, once again, they can hide in "the call of the tribe", whose concept is proposed by Karl Popper. This phenomenon is called "retribalization".

In other words, in the age of the Internet, we should have been more interconnected, but on the recommendation of AI algorithms, it may make people more closed from each other, not get closer to one another. Since the adaptive recommend framework used by Jinri Toutiao (a news App) and Facebook, usually uphold the principle of Matthew: "For unto everyone that hath shall be given and he shall have abundance." But is this really the "Gospel" for general public? This is an important issue that is worthy of deep consideration.

\section{Countermeasures and conclusions}

From the above analysis, we can see that in the era of big data, AI algorithms do indeed make us see the world of data more clearly. However, everything has two sides, because it may also bring about potential negative effects. The former Amazon Chief scientist Andreas Weigend presented us with a general principle: "Our lives should not be driven by data. They should be empowered by it." [6]

Part of the solutions to address many of those concerns have actually been written into the wisdom of our sages. For example, in order to relieve tendency of being a fatuous king in digital age, the ancient Chinese philosophy helps, "Listen to both sides and you will be enlightened; heed only one side and you will be benighted." More "listening" to several dimensions of "facts" independently, will bring a clearer fact.

For another example, the solution to both the generalization of quick thinking and Hegel's master-slave identity transformation are similar, they exit both due to over-reliance on AI algorithm. Therefore, independence, self-reliance is not only applicable to individuals and countries, but also applies to individuals in the era of big data. In this scenario, Occam's razor also works. "If not necessary, not by entity." This is because that, once one entity (such as AI algorithm) is involved in our life or work, then this object may give us an uncontrollable result. 
In addition, with the increasing maturity of blockchain technology, it also provides a technical perspective for us to solve retribalization. Blockchain is not an extension of the Internet, but a great subversion of the Internet. At present, each of us lives in a block (similar to a tribe) and is constrained by this small environment, and it is increasingly difficult to escape. If we can make innovations in the "chain" of the blockchain, break the barriers of the blocks and make the blocks link with each other efficiently, this will yield a huge dividend in the future.

AI will become IA (Intelligence Augmentation) if it is used properly. However, if used improperly, artificial unintelligence will happen and AI may become an elusive Leviathan (a primeval monster that symbolizes evil in Bible). In this regard, we are expected to have a cautious optimism, embrace the uncertainties of AI era [7], and form a cross-border harmony between artificial intelligence and human intelligence.

\section{References}

1. Hammond, K.J.: The Value of Big Data Isnt the Data. Harvard Business Review, https://hbr.org/2013/05/the-value-of-big-data-isnt-the. Last accessed 7 Aug 2018

2. Victor M.-S., Keneth C.:Big Data:A Revolution That Will Transform How We Live, Work, and Think. New York: Houghton Mifflin Harcourt (2013)

3. Wang, J., Yuan, S.: Real-time bidding: A new frontier of computational advertising research. In : Proceedings of the Eighth ACM International Conference on Web Search and Data Mining, pp. 415-416). ACM (2015)

4. Meredith B.: Artificial Unintelligence: How Computers Misunderstand the World. The MIT Press (2018)

5. Kahneman, D., P. Egan: Thinking, fast and slow. Farrar, Straus and Giroux New York (2011)

6. Weigend, A.S.: Data for the People: How to Make Our Post-Privacy Economy Work for You. Basic Books (2017)

7. Sam B., Chris W., Simon W.: Embrace the uncertainty of AI, McKinsey \& Company. https://www.mckinsey.com/business-functions/organization/ our-insights/the-organization-blog/embrace-the-uncertainty-of-ai. Last accessed 7 Aug 2018 